\title{
Powers of a rational number modulo 1 cannot lie in a small interval
}

by

\section{Artūras Dubickas (Vilnius)}

1. Introduction. Let throughout $\mathbb{R}, \mathbb{Z}$ and $\mathbb{N}$ be the sets of real numbers, integers and positive integers, respectively. We will denote by $[x]$ and $\{x\}$ the integral part and the fractional part of $x \in \mathbb{R}$, respectively. For an interval $[s, s+t) \subset[0,1)$ and two integers $p, q$, where $1<q<p$, put

$$
Z_{p / q}(s, s+t)=\left\{\xi \neq 0: s \leq\left\{\xi(p / q)^{n}\right\}<s+t \text { for all integer } n \geq 0\right\} .
$$

In [14] Mahler asked whether the set $Z_{3 / 2}(0,1 / 2)$ is empty or not. A hypothetical $\xi \in Z_{3 / 2}(0,1 / 2)$ is called a $Z$-number. It seems very likely that $Z$-numbers do not exist. An important step towards solution of this problem has been made by Flatto, Lagarias and Pollington [12] (see also [11]). It was proved in [12] that for coprime positive integers $p>q>1$ and any $\xi \neq 0$ the inequality

$$
\limsup _{n \rightarrow \infty}\left\{\xi(p / q)^{n}\right\}-\liminf _{n \rightarrow \infty}\left\{\xi(p / q)^{n}\right\} \geq 1 / p
$$

holds. A generalization of (1) to powers of algebraic numbers is given in [9]. The case of positive integers, namely, $p \geq 2, q=1$ was studied in [7].

Inequality (1) implies that the fractional parts $\left\{\xi(p / q)^{n}\right\}, n=0,1,2, \ldots$, cannot lie in an interval of length strictly smaller than $1 / p$. Can they all lie in an interval of length $1 / p$ ? This small step towards Mahler's problem turns out to be very difficult. It was shown in [12] that the set of $s \in[0,1-1 / p]$ for which $Z_{p / q}(s, s+1 / p)$ is empty is everywhere dense in $[0,1-1 / p]$. Naturally, it was conjectured that $Z_{p / q}(s, s+1 / p)$ is empty for each $s \in[0,1-1 / p]$ (see p. 138 in [12]).

This problem is still open, although Bugeaud has made some progress in this direction in [6]. He was able to prove that $Z_{p / q}(s, s+1 / p)$ is empty for almost all $s \in[0,1-1 / p]$. Moreover, he showed that the set $Z_{3 / 2}(s, s+1 / 3)$ 
is empty for

$$
s \in\{0\} \cup[8 / 57,4 / 19] \cup[4 / 15,2 / 5] \cup[26 / 57,10 / 19] \cup\{2 / 3\} .
$$

In this paper, we prove the set $Z_{p / q}(s, s+1 / p)$ to be indeed empty for each $s \in[0,1-1 / p]$ provided that $p, q$ are integers satisfying $1<q<p<q^{2}$. More precisely, we prove the following:

THeOREM 1. Let $p, q$ be two coprime integers satisfying $1<q<p<q^{2}$, and let $I$ be a closed subinterval of length $1 / p$ of the torus $\mathbb{R} / \mathbb{Z}$. Then for each real number $\xi \neq 0$ we have $\left\{\xi(p / q)^{n}\right\} \notin I$ for infinitely many $n \in \mathbb{N}$.

Of course, Theorem 1 implies that the set $Z_{p / q}(s, s+1 / p)$ is empty if $1<$ $q<p<q^{2}$ and $s \in[0,1-1 / p]$. In particular, the number $p / q=3 / 2$ satisfies the condition $p<q^{2}$. So the most interesting application of Theorem 1 is that the set $Z_{3 / 2}(s, s+1 / 3)$ is empty for every $s \in[0,2 / 3]$. This solves the problem considered in Corollary 1.4a of [12] and Corollary 1 of [6].

2. Auxiliary results. We shall need some terminology which is usually used in combinatorics on words (see, e.g., [2], [4], [13]). Any sequence (finite or infinite) of letters of an alphabet $A$ is called a word. Any string of consecutive letters of a word is called its factor. A string of letters starting from the first letter is called a prefix. Let $p(\mathbf{w}, m)$ be the number of distinct factors of length $m$ occurring in the word $\mathbf{w}$. By an old result of Morse and Hedlund [15], every infinite word $\mathbf{w}=w_{1} w_{2} w_{3} \ldots$ is either periodic (which means that there exist $n_{0}, t \in \mathbb{N}$ such that $w_{n+t}=w_{n}$ for every $\left.n \geq n_{0}\right)$ or $p(\mathbf{w}, m) \geq m+1$ for each $m \in \mathbb{N}$. Every infinite word which is not periodic is called aperiodic.

An infinite word $\mathbf{w}$ is called Sturmian if $p(\mathbf{w}, m)=m+1$ for every $m \in \mathbb{N}$. In particular, every Sturmian word is over two letters. Below we shall use the fact that an aperiodic word on the alphabet $A=\{U, V\}$ is Sturmian if and only if for any finite word $\mathbf{u}$ on $A$ either $U \mathbf{u} U$ or $V \mathbf{u} V$ is not a factor of $\mathbf{w}$.

Fix two relatively prime integers $p>q>1$ and two real numbers $\xi \neq 0$ and $\nu$. Set

$$
x_{n}=\left[\xi(p / q)^{n}+\nu\right] \text { and } y_{n}=\left\{\xi(p / q)^{n}+\nu\right\} .
$$

Let also

$$
s_{n}=q x_{n+1}-p x_{n} \quad \text { and } \quad t_{n}=-q y_{n+1}+p y_{n} .
$$

From $(p / q)\left(x_{n}+y_{n}-\nu\right)=x_{n+1}+y_{n+1}-\nu$ it follows that

$$
s_{n}=t_{n}-(p-q) \nu \text {. }
$$

Using $-q<t_{n}<p$ we derive that

$$
-q+(p-q) \nu<s_{n}<p-(p-q) \nu
$$


for each $n \geq 0$. Let

$$
\mathcal{A}=\mathcal{A}(p, q, \nu)=\mathbb{Z} \cap(-q+(p-q) \nu, p-(p-q) \nu) .
$$

With this notation, we have the following:

LEMMA 2. For relatively prime $p>q>1$ and arbitrary $\xi \neq 0$ and $\nu$, the word $\mathbf{w}=s_{0} s_{1} s_{2} s_{3} \ldots$ is an aperiodic word in the finite alphabet $\mathcal{A}$.

This is exactly Lemma 2 of [10]. (The fact that in [10] it is stated only for $\xi>0$ is irrelevant.) Lemma 2 combined with (3) implies that the word $t_{0} t_{1} t_{2} t_{3} \ldots$ is an aperiodic word on the finite alphabet $\mathcal{A}+(p-q) \nu$.

It is easy to see that, for any $m \in \mathbb{N}$, we have $x_{n+m}=(p / q) x_{n+m-1}+$ $s_{n+m-1} / q$. Expressing $x_{n+m-1}$ by $x_{n+m-2}$ and so on (up to $x_{n}$ ), we find that

$$
x_{n+m}=(p / q)^{m} x_{n}+\left((p / q)^{m-1} s_{n}+(p / q)^{m-2} s_{n+1}+\cdots+s_{n+m-1}\right) / q .
$$

Analogously, using $t_{n}=s_{n}+(p-q) \nu$ for $n \geq 0$, we derive that

$$
y_{n+m}=(p / q)^{m} y_{n}-\left((p / q)^{m-1} t_{n}+(p / q)^{m-2} t_{n+1}+\cdots+t_{n+m-1}\right) / q .
$$

Our next statement also holds for arbitrary coprime integers $p>q>1$, but $\nu$ will be selected in a special way:

TheOREM 3. Let $p>q>1$ be two coprime integers, and let $\xi \neq 0$ be a real number. Suppose $I=[s, s+1 / p](\bmod 1)$, where $0 \leq s<1$, is a closed subinterval of the torus $\mathbb{R} / \mathbb{Z}$ such that $\left\{\xi(p / q)^{n}\right\} \in I$ for each integer $n \geq 0$. Then the word $s_{0} s_{1} s_{2} s_{3} \ldots$, where

$$
s_{n}=q\left[\xi(p / q)^{n+1}-s\right]-p\left[\xi(p / q)^{n}-s\right]
$$

for $n \geq 0$, is a Sturmian word on the two-symbol alphabet $\{k, k+1\}$ for some $k \in \mathbb{Z}$.

Proof. Take $\nu=-s$. Then

$$
0 \leq y_{n}=\left\{\xi(p / q)^{n}+\nu\right\}=\left\{\xi(p / q)^{n}-s\right\} \leq 1 / p
$$

for each $n \geq 0$. Suppose first that the word $\mathbf{w}=s_{0} s_{1} s_{2} s_{3} \ldots$ contains at least three distinct letters (in this case simply integers). Then there exist $u, v \in \mathbb{Z}$ such that, for some $i, j \geq 0$, we have $s_{i}=u, s_{j}=v$ with $u+2 \leq v$. Then, using (2), (3) with $n=i$ and the inequalities $y_{i} \geq 0$ and $y_{i+1} \leq 1 / p$, we deduce that

$$
-u=-p y_{i}+q y_{i+1}+(p-q) \nu \leq q / p+(p-q) \nu .
$$

Similarly, from (2), (3) with $n=j$, we have

$$
v=p y_{j}-q y_{j+1}-(p-q) \nu \leq 1-(p-q) \nu .
$$

Adding these two inequalities, we obtain

$$
2 \leq v-u \leq q / p+(p-q) \nu+1-(p-q) \nu=q / p+1<2,
$$


which is impossible. Hence $\mathbf{w}$ must be a word on an alphabet of two letters which are consecutive integers, say, $k$ and $k+1$, where $k \in \mathbb{Z}$.

By Lemma 2, we already know that the word $\mathbf{w}$ is aperiodic. If $\mathbf{w}$ is not Sturmian, then there is a word $\mathbf{u}$ on the alphabet $\{k, k+1\}$ such that $(k+1) \mathbf{u}(k+1)$ and $k \mathbf{u} k$ are both factors of $\mathbf{w}$ (see, e.g., Proposition 2.1.3 and Theorem 2.1.5 in [13]). Suppose that the factors $k \mathbf{u} k$ and $(k+1) \mathbf{u}(k+1)$ of length $m$ start at the $i$ th and $j$ th places of $\mathbf{w}=s_{0} s_{1} s_{2} s_{3} \ldots$, where $i, j \geq 0$. Setting in (5) $n=j$ and $n=i$ and subtracting the second equality from the first, we obtain

$$
y_{j+m}-y_{i+m}=(p / q)^{m}\left(y_{j}-y_{i}\right)-\left((p / q)^{m-1}+1\right) / q .
$$

Since $y_{i}, y_{j+m} \geq 0$ and $y_{j}, y_{i+m} \leq 1 / p$, this implies that

$$
\left((p / q)^{m-1}+1\right) / q=(p / q)^{m}\left(y_{j}-y_{i}\right)-y_{j+m}+y_{i+m} \leq\left((p / q)^{m}+1\right) / p .
$$

Multiplying by $p$ we obtain $(p / q)^{m}+p / q \leq(p / q)^{m}+1$, i.e., $p \leq q$, a contradiction. Hence $\mathbf{w}$ must be a Sturmian word over the alphabet $\{k, k+1\}$.

3. Proof of Theorem 1. Suppose there is a closed subinterval $I=$ $[s, s+1 / p](\bmod 1)$, where $0 \leq s<1$, of the torus $\mathbb{R} / \mathbb{Z}$ and some $\xi \neq 0$ such that $\left\{\xi(p / q)^{n}\right\} \in I$ for each $n \geq n_{1}$. On replacing $\xi$ by $\xi(p / q)^{n_{1}}$, we can assume that $\left\{\xi(p / q)^{n}\right\} \in I$ for each integer $n \geq 0$. By Theorem 3, $\mathbf{w}=s_{0} s_{1} s_{2} s_{3} \ldots$, where

$$
s_{n}=q\left[\xi(p / q)^{n+1}-s\right]-p\left[\xi(p / q)^{n}-s\right]=q x_{n+1}-p x_{n}
$$

for $n \geq 0$, must be a Sturmian word on an alphabet $\{k, k+1\}$. Using (4) we will show that this is not the case.

Consider $m+2$ words of length $m$ each, namely, $s_{n} s_{n+1} \ldots s_{n+m-1}$, where $n=0, \ldots, m+1$. Since $\mathbf{w}$ is Sturmian, we have $p(\mathbf{w}, m)=m+1$, so at least two of these $m+2$ words must be equal, say, $s_{i} s_{i+1} \ldots s_{i+m-1}=$ $s_{j} s_{j+1} \ldots s_{j+m-1}$, where $0 \leq i=i(m)<j=j(m) \leq m+1$. Selecting in (4) first $n=i$ then $n=j$ and subtracting the first equality from the second, we obtain

$$
x_{m+j}-x_{m+i}=(p / q)^{m}\left(x_{j}-x_{i}\right) .
$$

Since $\xi \neq 0$, there is a positive integer $m_{0}$ such that the sequence $x_{m}=$ $\left[\xi(p / q)^{m}-s\right]$ is increasing for $m \geq m_{0}$ if $\xi>0$ or decreasing for $m \geq m_{0}$ if $\xi<0$. In both cases, the difference $x_{m+j}-x_{m+i}$ is nonzero for each $m \geq m_{0}$. Hence, by (6), we have $x_{j} \neq x_{i}$ for each $m \geq m_{0}$. Clearly, $x_{n} \in \mathbb{Z}$ for each integer $n \geq 0$, so (6) implies that the number $q^{m}$ divides the difference $x_{j}-x_{i}$. In particular,

$$
q^{m} \leq\left|x_{j}-x_{i}\right| \leq\left|x_{i}\right|+\left|x_{j}\right|
$$

because $x_{j} \neq x_{i}$. Recall that $s_{n} \in \mathcal{A}(p, q,-s)$, so $\left|s_{n}\right|<2 p$. Using (4) with $n=0$ we deduce that $\left|x_{i}\right| \leq c(p / q)^{i}$ and $\left|x_{j}\right| \leq c(p / q)^{j}$, where the constant $c$ 
is equal to, say, $\left|x_{0}\right|+2 p$. It follows that

$$
\begin{aligned}
q^{m} & \leq\left|x_{i}\right|+\left|x_{j}\right| \leq c(p / q)^{i}+c(p / q)^{j} \\
& \leq 2 c(p / q)^{m+1}=2 c(p / q)(p / q)^{m} \leq c p(p / q)^{m},
\end{aligned}
$$

because $i<j \leq m+1$. Taking $m$ th roots and using $p \leq q^{2}-1$, we derive that

$$
q^{2} \leq(c p)^{1 / m} p \leq(c p)^{1 / m}\left(q^{2}-1\right) .
$$

This is impossible if $m \geq m_{0}$ is so large that $(c p)^{1 / m}<1+1 / q^{2}$. This proves the theorem.

4. Concluding remarks. Note that Theorem 1 only holds under the condition $p<q^{2}$. The question of whether the set $Z_{p / q}(s, s+1 / p)$ is also empty if $p>q^{2}$ remains open. The condition $p<q^{2}$ can be made less restrictive in case the constant $I_{S}$ described below is greater than 2 .

Before giving a formal definition of the constant $I_{S}$, let us recall that every Sturmian word w (in this context more often called Sturmian sequence) begins in arbitrarily long squares, namely, there exist arbitrarily long prefixes of $\mathbf{w}$ of the form $\mathbf{v}^{2}$ (see, e.g., [1], [8]). Also, every Sturmian sequence contains arbitrarily long cubes, i.e., the prefixes of $\mathbf{w}$ are of the form $\mathbf{u v}^{3}$ with arbitrarily large $|\mathbf{v}|$ (see [3], [16]). Given a Sturmian sequence $\mathbf{w}$, we define $I(\mathbf{w})$ as

$$
I(\mathbf{w})=\sup _{\sigma \geq 0, \tau \geq 2} \frac{\tau+\sigma}{1+\sigma},
$$

where the supremum is taken over real numbers $\sigma \geq 0$ and $\tau \geq 2$ such that, for every positive integer $k$, there exist two words $\mathbf{u}_{k}$ and $\mathbf{v}_{k}$ satisfying the following three conditions:

- $\mathbf{u}_{k} \mathbf{v}_{k}^{\tau}$ is a prefix of $\mathbf{w}$ for each $k \in \mathbb{N}$,

- $\left|\mathbf{u}_{k}\right| \leq \sigma\left|\mathbf{v}_{k}\right|$ for each $k \in \mathbb{N}$,

- $\left|\mathbf{v}_{k}\right| \rightarrow \infty$ as $k \rightarrow \infty$.

Throughout, $\mathbf{v}^{\tau}$ is defined as a word of length $[\tau|\mathbf{v}|]$ consisting of $[\tau]$ words $\mathbf{v}$ and the prefix of $\mathbf{v}$ of length $[\{\tau\}|\mathbf{v}|]$. The constant $I_{S}$ is then defined by the formula

$$
I_{S}=\inf _{\mathbf{w} \text { Sturmian }} I(\mathbf{w}) .
$$

By the above-mentioned results, one can select $\sigma=0$ and $\tau=2$ for every Sturmian sequence $\mathbf{w}$, hence $I_{S} \geq 2$. We do not know whether $I_{S}$ is strictly greater than 2 or not. However, the result of Berthé, Holton and Zamboni [5] implies that $\tau$ must be at most 3 for some Sturmian sequences $\mathbf{w}$. For those $\mathbf{w}$ we have $I(\mathbf{w}) \leq 3$, hence $I_{S} \leq 3$. Consequently, $2 \leq I_{S} \leq 3$. 
We claim that the conclusion of Theorem 1 holds for any coprime integers $p, q$ satisfying $1<q<p<q^{I_{S}}$. Our argument follows the same line as that in the proof of Theorem 1. It is based on Theorem 3 and repeated application of (4).

Indeed, suppose that two integers $p, q$ satisfy $1<q<p<q^{I_{S}}$. As we already observed above, $I_{S} \leq 3$, so $I_{S}<\infty$. Fix $\varepsilon>0$ so small that $p<q^{I_{S}-3 \varepsilon}$ and fix two real numbers $\sigma \geq 0, \tau \geq 2$ for which the above three conditions are satisfied for a Sturmian word $\mathbf{w}=s_{0} s_{1} s_{2} s_{3} \ldots$ and $(\tau+\sigma) /(1+\sigma)>I(\mathbf{w})-\varepsilon$. Here $s_{n}, n=0,1,2, \ldots$, and $\nu=-s$ are defined as in Theorem 3. Note that $I(\mathbf{w}) \geq I_{S}$, so $(\tau+\sigma) /(1+\sigma)>I_{S}-\varepsilon$. We will show that this is impossible.

Set $\mathbf{w}_{k}=\mathbf{v}_{k}^{\{\tau\}}$, so that $\mathbf{v}_{k}^{\tau}=\mathbf{v}_{k}^{[\tau]} \mathbf{w}_{k}$. Note that $\mathbf{u}_{k} \mathbf{v}_{k}^{[\tau]-1} \mathbf{w}_{k}$ and $\mathbf{u}_{k} \mathbf{v}_{k}^{[\tau]} \mathbf{w}_{k}$ $=\mathbf{u}_{k} \mathbf{v}_{k} \mathbf{v}_{k}^{[\tau]-1} \mathbf{w}_{k}$ both are prefixes of $\mathbf{w}$. So the words $s_{i} s_{i+1} \ldots s_{i+m-1}$ and $s_{j} s_{j+1} \ldots s_{j+m-1}$, where $i=\left|\mathbf{u}_{k}\right|, j=\left|\mathbf{u}_{k}\right|+\left|\mathbf{v}_{k}\right|$ and $m=\left|\mathbf{v}_{k}^{[\tau]-1} \mathbf{w}_{k}\right|$, are equal. Selecting in (4) first $n=i$ then $n=j$ and subtracting the first equality from the second, we obtain $x_{m+j}-x_{m+i}=(p / q)^{m}\left(x_{j}-x_{i}\right)$ as in (6). As above, $q^{m} \mid\left(x_{j}-x_{i}\right)$, where $x_{j}-x_{i}$ is a nonzero integer for $m$ large enough. From (4) it follows that $q^{m} \leq\left|x_{j}\right|+\left|x_{i}\right|<c_{1}(p / q)^{j}$, giving $q^{m+j}<c_{1} p^{j}$ with some positive number $c_{1}=c_{1}(p, q, \xi,-s)$. Hence $q^{m / j+1}<c_{1}^{1 / j} p$.

By the definition of $\mathbf{u}_{k}$ and $\mathbf{v}_{k}$, we have

$$
\begin{aligned}
\frac{m}{j} & =\frac{\left|\mathbf{v}_{k}^{[\tau]-1} \mathbf{w}_{k}\right|}{\left|\mathbf{u}_{k}\right|+\left|\mathbf{v}_{k}\right|}=\frac{([\tau]-1)\left|\mathbf{v}_{k}\right|+\left[\{\tau\}\left|\mathbf{v}_{k}\right|\right]}{\left|\mathbf{u}_{k}\right|+\left|\mathbf{v}_{k}\right|} \\
& >\frac{(\tau-1)\left|\mathbf{v}_{k}\right|-1}{\left|\mathbf{u}_{k}\right|+\left|\mathbf{v}_{k}\right|} \geq \frac{(\tau-1)\left|\mathbf{v}_{k}\right|-1}{\sigma\left|\mathbf{v}_{k}\right|+\left|\mathbf{v}_{k}\right|} .
\end{aligned}
$$

Hence $m / j \geq(\tau-1) /(1+\sigma)-\varepsilon$ for $k$ large enough, because $1 /\left|\mathbf{v}_{k}\right| \rightarrow 0$ as $k \rightarrow \infty$. Adding 1 to both sides gives

$$
m / j+1 \geq(\tau+\sigma) /(1+\sigma)-\varepsilon>I_{S}-2 \varepsilon .
$$

Therefore $q^{m / j+1}>q^{I_{S}-2 \varepsilon}$. It follows that $q^{I_{S}-2 \varepsilon}<c_{1}^{1 / j} p<c_{1}^{1 / j} q^{I_{S}-3 \varepsilon}$, so $q^{\varepsilon}<c_{1}^{1 / j}$. Clearly, if $k \rightarrow \infty$, then $\left|\mathbf{v}_{k}\right| \rightarrow \infty$, so $m \rightarrow \infty$ and $j \rightarrow \infty$. By taking $k$ so large that $c_{1}^{1 / j}<1+\varepsilon / 2$, we deduce that $q^{\varepsilon}<1+\varepsilon / 2$. However, this is impossible, because $q^{\varepsilon}$ is greater than $1+\varepsilon \log q \geq 1+\varepsilon \log 2>$ $1+0.69 \varepsilon$. This proves our assertion.

Acknowledgements. I thank a referee for very careful reading of the manuscript and pointing out several inaccuracies.

This research was partially supported by the Lithuanian State Science and Studies Foundation. 


\section{References}

[1] J.-P. Allouche, J. P. Davison, M. Queffélec and L. Q. Zamboni, Transcendence of Sturmian or morphic continued fractions, J. Number Theory 91 (2001), 39-66.

[2] J.-P. Allouche and J. Shallit, Automatic Sequences. Theory, Applications, Generalizations, Cambridge Univ. Press, Cambridge, 2003.

[3] J. Berstel, On the index of Sturmian words, in: Jewels are Forever. Contributions on Theoretical Computer Science in Honor of Arto Salomaa, J. Karhumäki et al. (eds.), Springer, Berlin, 1999, 287-294.

[4] J. Berstel and J. Karhumäki, Combinatorics on words - a tutorial, in: Current Trends in Theoretical Computer Science. The Challenge of the New Century. Vol. 2: Formal Models and Semantics, G. Păun et al. (eds.), World Scientific, River Edge, NJ, 2004, 415-475.

[5] V. Berthé, C. Holton and L. Q. Zamboni, Initial powers of Sturmian sequences, Acta Arith. 122 (2006), 315-347.

[6] Y. Bugeaud, Linear mod one transformations and the distribution of fractional parts $\left\{\xi(p / q)^{n}\right\}$, ibid. $114(2004), 301-311$.

[7] Y. Bugeaud and A. Dubickas, Fractional parts of powers and Sturmian words, C. R. Math. Acad. Sci. Paris 341 (2005), 69-74.

[8] D. Damanik, R. Killip and D. Lenz, Uniform spectral properties of one-dimensional quasicrystals, III. $\alpha$-continuity, Comm. Math. Phys. 212 (2000), 191-204.

[9] A. Dubickas, Arithmetical properties of powers of algebraic numbers, Bull. London Math. Soc. 38 (2006), 70-80.

[10] A. Dubickas and A. Novikas, Integer parts of powers of rational numbers, Math. Z. 251 (2005), 635-648.

[11] L. Flatto, $Z$-numbers and $\beta$-transformations, in: Symbolic Dynamics and Its Applications (New Haven, CT, 1991), P. Walters (ed.), Contemp. Math. 135, Amer. Math. Soc., Providence, RI, 1992, 181-201.

[12] L. Flatto, J. C. Lagarias and A. D. Pollington, On the range of fractional parts $\left\{\xi(p / q)^{n}\right\}$, Acta Arith. 70 (1995), 125-147.

[13] M. Lothaire, Algebraic combinatorics on words, Encyclopedia Math. Appl. 90, Cambridge Univ. Press, Cambridge, 2002.

[14] K. Mahler, An unsolved problem on the powers of 3/2, J. Austral. Math. Soc. 8 (1968), 313-321.

[15] M. Morse and G. A. Hedlund, Symbolic dynamics II: Sturmian trajectories, Amer. J. Math. 62 (1940), 1-42.

[16] D. Vandeth, Sturmian words and words with a critical exponent, Theoret. Comput. Sci. 242 (2000), 283-300.

Department of Mathematics and Informatics

Vilnius University

Naugarduko 24

Vilnius LT-03225, Lithuania

E-mail: arturas.dubickas@mif.vu.lt

Received on 12.3.2008

and in revised form on 19.10.2008 